\title{
Skeletal muscle-derived extracellular vesicles are altered with chronic contractile activity
}

Patience O. Obi ${ }^{1,3,4,8,9}$, Samira Seif $1^{1,3,8,9}$, Benjamin Bydak ${ }^{1,3,8,9}$, Taiana M. Pierdoná1 ${ }^{13,8,9}$, Emily TurnerBrannen ${ }^{3,5,8}$, Adrian R. West ${ }^{3,5,8}$, Hagar I. Labouta ${ }^{6,8}$, Joseph W. Gordon ${ }^{1,2,3,7,8}$, and Ayesha Saleem ${ }^{1,2,3,4,8,9}$

${ }^{1}$ Diabetes Research Envisioned and Accomplished in Manitoba (DREAM) Research Theme, Winnipeg, MB, Canada

${ }^{2}$ DEVOTION Research Cluster, Winnipeg, MB, Canada

${ }^{3}$ Biology of Breathing Research Theme, Winnipeg, MB, Canada

${ }^{4}$ Applied Health Sciences, University of Manitoba, Winnipeg, MB, Canada

${ }_{5}^{5}$ Department of Physiology and Pathophysiology, Rady Faculty of Health Sciences, University of Manitoba, Winnipeg, MB. Canada

${ }^{6}$ College of Pharmacy, Rady Faculty of Health Sciences, University of Manitoba, Winnipeg, MB

${ }^{7}$ College of Nursing, Rady Faculty of Health Sciences, University of Manitoba, Winnipeg, MB, Canada

${ }^{8}$ Children's Hospital Research Institute of Manitoba (CHRIM), Winnipeg, MB, Canada

${ }^{9}$ Faculty of Kinesiology and Recreation Management, University of Manitoba, Winnipeg, MB, Canada

* To whom correspondence should be addressed:

Ayesha Saleem, PhD

Assistant Professor, Faculty of Kinesiology and Recreation Management

University of Manitoba, Winnipeg, MB, R3T 2N2

120 Frank Kennedy Centre I 204-474-7617 I ayesha.saleem@umanitoba.ca

Research Scientist, Children's Hospital Research Institute of Manitoba (CHRIM)

John Buhler Research Centre (JBRC), Winnipeg, MB R3E 3P4

600A - 715 McDermot Avenue I 204-789-3688 I asaleem@chrim.ca

\section{Running head: Skeletal muscle EVs with chronic exercise}

Keywords: extracellular vesicles, exosomes, skeletal muscle, myoblasts, myotubes, differentiation, differential ultracentrifugation, chronic contractile activity 


\begin{abstract}
Extracellular vesicles (EVs) are small lipid membrane-bound structures that are secreted by all cells, and play a central role in cellular communication. EVs are released from skeletal muscle during exercise, but the effects of contractile activity on skeletal muscle-derived EVs (Skm-EVs) are poorly understood due to the challenges in distinguishing Skm-EVs derived from exercising muscle in vivo. To specifically characterize Skm-EVs, C2C12 myoblasts were differentiated into myotubes, and electrically paced (3h/day x 4days @14V, C-PACE EM, IonOptix) to mimic chronic exercise in vitro. EVs were isolated from conditioned media from control and stimulated myotubes using differential ultracentrifugation. Isolated EVs were characterized biophysically (size, zeta potential, yield, protein markers and by transmission electron microscopy; TEM). Chronic stimulation increased markers of mitochondrial biogenesis such as MitoTracker Red staining, cytochrome $c$ oxidase activity and expression of cytochrome $c(p<0.05, N=7-8)$ in stimulated vs. non-stimulated myotubes. The average size of EVs from chronically stimulated myotubes (CS-EVs, $132 \mathrm{~nm}$ ) was 26\% smaller than control (CON-EVs, $178 \mathrm{~nm})(\mathrm{p}<0.05, \mathrm{~N}=8)$. Size distribution analysis revealed that CS-EVs were enriched with 100-150 nm sized small EVs, while CONEVs were largely composed of $200-250 \mathrm{~nm}$ sized vesicles $(\mathrm{p}<0.05$, main interaction effect, $\mathrm{N}=8$ ). TEM confirmed the presence of round-shaped vesicles of about 30-100 $\mathrm{nm}$ with an intact lipid bilayer in CONEVs and CS-EVs. Zeta potential was $27 \%$ lower in CS-EVs vs. CON-EVs ( $<<0.05, \mathrm{~N}=8$ ), and total EV protein yield remained unchanged between groups. Protein-based EV characterization showed that both CON-EVs/CS-EVs were CD81+ but CD63-, and expressed hallmark cytosolic proteins recovered in EVs: Tsg101, Flotillin-1, HSP70, and Alix. CD81 and HSP70 expression increased in CS-EVs vs. CON-EVs $(\mathrm{p}<0.05, \mathrm{~N}=9)$. We evaluated if chronic stimulation affected whole cell expression of transmembrane and cytosolic proteins used in EV origin/purity analysis. CD63 and ApoA1 were reduced with chronic stimulation in myotube lysates $(\mathrm{p}<0.05, \mathrm{~N}=7$ ), whereas Tsg101, CD81, Flotillin-1 and HSP70 levels remained constant. Taken together, our study revealed that chronic stimulation triggers the release of more stable, smaller sized EVs, enriched with transmembrane and cytosolic protein markers of small EVs, and are CD81+/CD63- indicating the origin of these EVs might be ectosomal rather than endosomal. The upstream signaling cascades that regulate biogenesis of EVs with chronic stimulation, and whether skeletal muscle-EVs are released through endosomal or ectosomal pathways remains to be elucidated. Our findings support the role of chronic contractile activity in the modulation of EV biophysical characteristics. Whether this affects biological cargo recruitment into CS-EVs, and their subsequent biological activity remains to be established.
\end{abstract}




\section{Introduction}

Extracellular vesicles (EVs) play a central role in cell-cell communication. EVs are found in all biological fluids including blood, urine, cerebrospinal fluid, saliva, breast milk, amniotic fluid, among others [1], [2]. EVs have been traditionally divided into three subtypes based on their biogenesis, size, and biological properties. They include exosomes $(30-150 \mathrm{~nm})$ formed by the invagination of endosomal membranes leading to the formation of intraluminal vesicles enclosed within mutivesicular bodies (MVBs) that are subsequently exocytosed through the plasma membrane; microvesicles $(100-1000 \mathrm{~nm})$ formed by the outward budding of the plasma membrane, and apoptotic bodies $(500-5000 \mathrm{~nm})$ formed from the outward blebbing of an apoptotic cell membrane [3]. The Minimal Information for Studies of EVs (MISEV) guidelines recommend classifying EVs based on i) size, where small EVs (sEVs) are $<200 \mathrm{~nm}$ and medium/large EVs (m/lEVs) $>200 \mathrm{~nm}$, ii) biochemical composition, and iii) cell of origin [4]. EVs contain biological cargo such as DNA, mRNA, miRNA, proteins, lipids, and metabolites and can transfer them from parent to recipient cells [5]. In the last decades, EVs have been extensively studied in a host of diseases as potential biomarkers [6]-[10] and novel therapeutics for drug delivery [11]-[13]. More recently, researchers have been begun to investigate the role of EVs in intercellular communication during physiological challenges.

Regular exercise is an important physiological stressor that has a plethora of positive systemic benefits such as improved mitochondrial capacity, enhanced antioxidant profile, protection against cardiovascular diseases, and improved muscle strength, among many other beneficial adaptations [14][16]. Exercise affects almost all the tissues in the body but the key organ systems that beneficially adapt include skeletal muscle, adipose tissue, brain, bone marrow, heart, gut, pancreas, and liver [17]. Skeletal muscle is the largest organ system in the body, accounting for $40 \%$ of body mass, and is a critical regulator of whole body metabolic capacity. Strong evidence suggests that the systemic adaptative effects of exercise are potentiated in part by the release of factors known as myokines during exercise from skeletal muscle [15], [18]. Myokines are usually proteins that can travel to different organs in the body and act in an autocrine, paracrine or endocrine manner. Examples include interleukin (IL)-6, IL-10, IL-15, irisin, meteorin, myostatin, among others [18]. In addition to myokines, EVs can also be released from the skeletal muscle during exercise. There is growing interest in how acute and chronic exercise affects EVs and whether they play a role in mediating the benefical systemic effects of exercise [19]-[22].

Previous studies have shown that acute/chronic exercise in humans, mice and rats increases the release of EVs in the circulation, and modifies cargo componenents [23]-[30]. However, the majority of this work has been done using EVs isolated from whole blood. EVs in blood are comprised of a heterogenous mixture of vesicles derived from platelets, erythrocytes, endothelial cells, and other circulating and neighboring cells [24], [25]. Given the challenge in differentiating skeletal muscle-derived EVs (Skm-EVs) in systemic EV preparations, and the fact that skeletal muscle is 1) the major tissue system involved in muscle contraction during physical activity, 2) can modify paracrine and exocrine tissue/organ metabolism through release of myokines directly or packaged in EVs, and 3) accounts for 
$\sim 40 \%$ of the body mass, it is critical to distill the the impact of chronic exercise on the characteristics of Skm-EVs specifically.

In this study, we used chronic electrical stimulation (IonOptix C-PACE EM) to mimic exercise in vitro. While chronic electrical pulses stimulation of differentiated skeletal muscle myotubes (immature fibers) may not recapitulate all facets of endurance exercise training, it is a validated and well-established model to study the role of skeletal muscle in exercise-mediated adaptations such as mitochondrial biogenesis. Indeed chronic contractile activity can evoke similar adaptations in mitochondrial content/function as weeks of exercise training in vivo [31], [32]. Using this well-established model, we evaluated the effect of chronic stimulation on the release and biophysical characteristics of Skm-EVs. Murine $\mathrm{C} 2 \mathrm{C} 12$ skeletal muscle myoblasts were differentiated into myotubes, electrically paced ( $3 \mathrm{~h} /$ day $\mathrm{x}$ 4 days, $14 \mathrm{~V}, 1 \mathrm{~Hz}$ ), and EVs isolated from conditioned media of control and chronically stimulated cells by differential ultracentrifugation ( $\mathrm{dUC}$ ) as detailed previously [33]. Isolated EVs were characterized by dynamic light scattering (DLS, ZetaPALS) technology to determine biophysical properties and by Western blotting to evaluate any differences in chronically stimulated EVs (CS-EVs) vs.control EVs (CON-EVs). 


\section{Methods}

\section{Cell culture}

Murine $\mathrm{C} 2 \mathrm{C} 12$ myoblasts were seeded in a six well plate pre-coated with $0.2 \%$ gelatin, and grown in fresh Dulbecco's Modification of Eagle's Medium (DMEM; Sigma-Aldrich) supplemented with 10\% fetal bovine serum (FBS; Gibco/ThermoFisher Scientific) and 1\% penicillin/streptomycin (P/S) (growth media). Cells were grown at $37{ }^{\circ} \mathrm{C}$ in $5 \% \mathrm{CO} 2$ incubator for $24 \mathrm{~h}$. When myoblasts reached approximately $90-95 \%$ confluency, the growth media was switched to differentiation media (DMEM supplemented with 5\% heat-inactivated horse serum (HI-HS; Gibco/ThermoFisher Scientific) and 1\% P/S) for 5 days to differentiate myoblasts into immature muscle fibers, or myotubes. The overall experimental design and worklow is illustrated in Fig. 1.

\section{Chronic electrical stimulation of myotubes}

Chronic electrical pulse stimulation experiments were performed as previously described by Uguccioni and Hood [31] with some modifications. After five days of differentiation, myotubes were divided into control (CON) and chronically stimulated (CS) groups. The CS plate was stimulated using the C-Pace EM Cell culture stimulator, with C-dish and carbon electrodes (IonOptix, Milton, MA, United States) while the CON plate had the C-dish placed on top but without the carbon electrodes. The stimulation protocol was performed at a frequency of $1 \mathrm{~Hz}$ (at $2 \mathrm{~ms}$ pulses) and an intensity of $14 \mathrm{~V}$ chronically for $3 \mathrm{~h} /$ day for four consecutive days. After each bout of contractile activity, spent media was discarded and fresh differentiation media added to each well. This is important as previous observations by Saleem and Hood (unpublished, PhD dissertation) indicate that changing spent media post-stimulation improves protein and RNA yield from CS myotubes. Myotubes were left to recover for $21 \mathrm{~h}$ before the next bout of stimulation. The total stimulation protocol lasted for 4 days and immediately after the last day of stimulation, media was changed to exosome-depleted differentiation media (DMEM supplemeted with $5 \%$ exosome-depleted HI-HS and 1\% P/S) for both groups and myotubes left to recover for $21 \mathrm{~h}$. Exosomedepleted horse serum was used to remove any confounding effects of EVs found naturally in horse serum. Horse serum was depleted of exosomes/sEVs by spinning $10 \%$ horse serum at $100,000 \mathrm{xg}$ for $18 \mathrm{~h}$ before making differentiation media. $21 \mathrm{~h}$ after recovery, conditioned media from CON and CS myotubes was collected and used for EV isolation. Lastly, myotubes were harvested for protein extraction and Western blotting.

\section{Isolation of EVs by differential centrifugation}

EV isolation by dUC was performed according to the protocol by Théry et al. [33]. Briefly, conditioned media from each six well plate $(12 \mathrm{ml})$ was collected and centrifuged at $300 x g$ for $10 \mathrm{~min}$ at $4{ }^{\circ} \mathrm{C}$ to pellet dead cells (Sorvall ${ }^{\text {TM }}$ RC 6 Plus Centrifuge, F13-14 fixed angle rotor), followed by centrifugation at 2000xg for $10 \mathrm{~min}$ at $4{ }^{\circ} \mathrm{C}$ to remove cell debris. The resulting supernatant was then centrifuged at $10,000 \mathrm{xg}$ for 30 min at $4{ }^{\circ} \mathrm{C}$ to remove large vesicles. Using an ultracentrifuge (Sorvall ${ }^{\mathrm{TM}}$ MTX 150 Micro-Ultracentrifuge, S58-A fixed angle rotor), the supernantant was spun at $100,000 x \mathrm{~g}$ for $70 \mathrm{~min}$ at $4{ }^{\circ} \mathrm{C}$ to obtain the exosome/small EV pellet, after which the resulting supernatant, labelled as sEV-depleted conditioned media was collected and stored for later use. The exosome/sEV pellet was resuspended in $1 \mathrm{~mL}$ PBS and 
centrifuged again at $100,000 \mathrm{xg}$ for $70 \mathrm{~min}$ at $4{ }^{\circ} \mathrm{C}$. After centrifugation, the final exosome/sEV pellet was resuspended in $50 \mu \mathrm{L}$ PBS and used for subsequent analysis.

\section{Dynamic light scattering (DLS)}

Measurements of EV size and zeta potential were performed by phase analysis DLS using a NanoBrook ZetaPALS (Brookhaven Instruments, Holtsville, NY, USA) in collaboration with Dr. Hagar Labouta's lab (College of Pharmacy, University of Manitoba). EV samples were diluted 1:75 with $0.22 \mu \mathrm{m}$ filtered PBS. Five measurements were recorded for each sample with a dust cut-off set to 40 . EV size was measured as an intensity averaged multimodal distribution using a scattering angle of $90^{\circ}$, and size bins were used to represent total size intensity within a given size range. Zeta potential analysis was performed using a Solvent-Resistant Electrode (NanoBrook) and BI-SCGO $4.5 \mathrm{ml}$ cuvettes (NanoBrook). Smoluchowski formula was used to calculate zeta potential and final results were averaged irrespective of the charge (negative/positive) [34]. All measurements were performed in PBS (pH 7.4) at $25^{\circ} \mathrm{C}$.

\section{Transmission electron microscopy (TEM)}

Freshly isolated EVs $(10 \mu \mathrm{l})$ were fixed with equal volume of $4 \%$ paraformaldehyde and carefully applied onto a copper grid for $20 \mathrm{~min}$ at room temperature. Grids were washed with $100 \mu \mathrm{PBS}$ and fixed with $1 \%$ glutaraldehyde for $5 \mathrm{~min}$. To remove the excess fixation buffer, the grids were washed 8 times with $100 \mu$ distilled water, after which they were negatively stained with $1 \%$ uranyl acetate for 2 min and left to airdry at room temperature. TEM grids were visualized using Phillips CM10 Electron Microscope operated at $60 \mathrm{kV}$ using the fee-for-service at the Histology core facility, Department of Human Anatomy and Cell Science, University of Manitoba.

\section{EV protein extraction, concentration and yield}

EV preparations were lysed using 1:1 Pierce RIPA solution with protease inhibitor tablet (Roche) and EV concentration was determined using Pierce ${ }^{\mathrm{TM}}$ MicroBCA protein assay kit (ThermoFisher Scientific) following the manufacturer's instructions. Briefly, the working reagent was prepared by mixing 25 parts of Reagent A, 24 parts of Reagent B and 1 part of Reagent C as detailed before [35]. Standards were prepared by serial dilution of $2 \mathrm{mg} / \mathrm{ml}$ bovine serum albumin (BSA) ampule into clean vials using ultrapure water. $150 \mu \mathrm{L}$ of each standard or sample was added to a 96-well microplate in duplicates, followed by the addition of $150 \mu \mathrm{L}$ of working reagent to each well. Samples were incubated $37^{\circ} \mathrm{C}$ for $2 \mathrm{~h}$ and absorbance was measured at $562 \mathrm{~nm}$ using a microplate spectrophotometer (BioTek, Epoch). Protein yield was determined by multiplying the protein concentration with the volume of EV lysate.

\section{MitoTracker Red staining}

MitoTracker Red stains mitochondria in live cells and is an acceptable marker of mitochondrial mass [32], [36]. After $21 \mathrm{~h}$ recovery post-last bout of contractile activity, myotubes were incubated with MitoTracker Red CMXRos (Cell Signalling Technology) and Hoechst 33258 (Sigma-Aldrich) at final concentrations of $50 \mathrm{nM}$ and $3 \mu \mathrm{g} / \mathrm{ml}$ respectively under normal culture conditions for $30 \mathrm{~min}$. Following incubation, cells were washed with PBS, and medium was switched to differentiation medium. Myotubes were visualized using the Olympus IX70 inverted microscope (Toronto, ON, Canada) with NIS Elements AR 3.0 software (Nikon Instruments Inc., Melville, NY, USA). Three representative images per well were taken, and the 
fluorescence intensity was quantified using ImageJ software (NIH, Bethesda, MD, USA), where the free hand tool was used to draw the region of interest (ROI) from at least three myotubes for each representative image, and mean intensity for each was averaged to get the total fluorescence intensity.

\section{Myotube enzyme extracts, protein concentration and yield}

Myotube whole cell enzyme extracts were harvested as previously described by Uguccioni and Hood [31] with some modifications. Myotubes were harvested $21 \mathrm{~h}$ after the last stimulation process using a cell scraper (Corning $\left.{ }^{\circledR}\right)$, resuspended in $50 \mu \mathrm{L}$ of enzyme extraction buffer (100 mM Na-K-phosphate, $2 \mathrm{mM}$ EDTA, pH 7.2), sonicated $3 \times 3$ secs on ice, subjected to repeated freeze-thaw cycles, vortexed vigorously for 5 secs and centrifuged at 14,000 rpm. The supernate was collected and used to measure protein concentration and yield, COX enzyme activity and Western blotting.

Protein concentration of control and stimulated myotube whole cell lysates was determined using Pierce $^{\mathrm{TM}}$ BCA protein assay kit (ThermoFisher Scientific) following the manufacturer's instructions as described before [37]. Briefly, a serial dilution of $2 \mathrm{mg} / \mathrm{ml}$ bovine serum albumin (BSA) in sterile water was prepared and used as standard. $25 \mu \mathrm{l}$ of each standard or sample was added in duplicates into a 96well microplate, followed by adding $200 \mu \mathrm{L}$ of the working reagent to each well. Samples were incubated at $37{ }^{\circ} \mathrm{C}$ for $30 \mathrm{~min}$ and the absorbance was measured at $562 \mathrm{~nm}$ using a microplate spectrophotometer (BioTek, Epoch). Protein yield was determined by multiplying the protein concentration with the volume of cell lysate.

\section{COX activity}

We measured mitochondrial cytochrome $c$ oxidase (COX) activity, a gold standard marker of mitochondrial biogenesis, in whole myotube whole cell enzyme extracts as previously described [38]. Briefly, enzyme extracts were added to a test solution containing fully reduced cytochrome $c$ (SigmaAldrich). COX activity was determined as the maximal rate of oxidation of fully reduced cytochrome $c$ measured by the change in absorbance over time at $550 \mathrm{~nm}$ using an imaging multi-mode reader (BioTek, Cytation 5) at $30{ }^{\circ} \mathrm{C}$. Protein concentration was determined using the Pierce ${ }^{\mathrm{TM}} \mathrm{BCA}$ protein assay kit as described above, and COX activity was normalized by total protein content.

\section{Western blotting}

$5 \mu \mathrm{g}$ of total protein from EVs or myotube whole cell extracts were resolved on a 12\% SDS-PAGE gel and subsequently transferred to nitrocellulose membranes. Membranes were then blocked for $1 \mathrm{~h}$ with $5 \%$ skim milk in $1 \times$ Tris-buffered saline-Tween 20 solution (TBST) at room temperature, followed by incubation with primary antibodies in $1 \%$ skim milk overnight at $4{ }^{\circ} \mathrm{C}$. The following primary antibodies were used: rabbit polyclonal anti-TSG101 (T5701, Sigma-Aldrich, 1:1000), rabbit polyclonal anti-CD63 (SAB4301607, Sigma-Aldrich, 1:200), mouse monoclonal anti-CD81 (sc-166029, Santa Cruz Biotechnology, 1:200), rabbit polyclonal anti-Flotillin-1 (F1180, Sigma-Aldrich, 1:200), mouse monoclonal anti-heat shock protein 70 (HSP70) (H5147, Sigma-Aldrich, 1:500), rabbit monoclonal anti-Alix (MCA2493, BioRad Laboratories, 1:200), mouse monoclonal anti-Apolipoprotein A1 (ApoA1) (0650-0050, Bio-Rad Laboratories, 1:200), rabbit polyclonal anti-cytochrome $c$ (AHP2302, Bio-Rad Laboratories, 1:500), and mouse monoclonal anti- $\beta$-actin (A5441-.2ML, Sigma-Aldrich, 1:5000). Subsequently, membranes were washed three times for 5 min with TBST, followed by incubation with anti-mouse (A16017, Thermofisher) 
or anti-rabbit (A16035, Thermofisher) IgG horseradish peroxidase secondary antibody $(1: 1,000-10,000)$ in $1 \%$ skim milk for $1 \mathrm{~h}$ at room temperature, after which the membrane is washed again three times for 5 min each with TBST. Membranes were developed using enhanced chemiluminescence detection reagent (Bio-Rad Laboratories), and the films were scanned using the ChemiDoc ${ }^{\mathrm{TM}}$ MP Imaging System (Bio-Rad Laboratories). The intensity of bands was quantified using the Image Lab Software (Bio-Rad Laboratories) and corrected for loading using coomassie blue or ponceau S staining.

\section{Statistical analysis}

All data were analyzed using an unpaired Student's t-test, except for size-distribution analysis for which we used a two-way repeated measures ANOVA. Multiple comparisions in the two-way ANOVA were corrected using Sidak's post hoc test. Individual data points are plotted, with mean \pm standard error of mean (SEM) shown as applicable. All graphs were created using GraphPad-Prism software (version 9.2.0). $\mathrm{p}$ values $\leq 0.05$ were considered significant. An $\mathrm{N}=6-9$ was conducted for all experiments. 


\section{Results}

\section{Chronic stimulation increases markers of mitochondrial biogenesis in C2C12 myotubes}

To validate our model of chronic contractile activity, several markers of mitochondrial biogenesis were examined. Control and chronically stimulated myotubes were stained with $50 \mathrm{nM}$ Mito Tracker CMXRos for $30 \mathrm{~min}$ at $37^{\circ} \mathrm{C}$ after conditioned media was collected for EV isolation. Representative fluorescent images at 40X and 10X are shown for CON and CS myotubes (Fig. 2A). Quantification of MitoTracker Red staining showed $9 \%$ increase in CS $v$ s. CON myotubes $(\mathrm{N}=8, \mathrm{p}<0.05$, Fig. 2B). Next, we measured COX activity in CON vs. CS myotubes, and measured a $\sim 51 \%$ increase in COX activity with chronic stimulation, indicative of an increase in mitochondrial biogenesis $(\mathrm{N}=8, \mathrm{p}<0.05$, Fig. $2 \mathrm{C})$ as previously detailed [31], [39]. We also evaluated the expression of cytochrome $c$ (Cyt C; a mitochrondrial protein important in electron transport chain) in CS vs. CON myotubes using Western blotting. CS myotubes showed a 2.2-fold increase in expression of Cyt $C$ relative to $C O N$ myotubes $(N=7, p<0.01$, Fig. 2D). Lastly, we measured protein yield, which was unchanged between groups ( $N=8$, Fig. 2E).

\section{Chronic stimulation alters EVs biophysical characteristics}

To evaluate the effects of chronic stimulation on Skm-EVs, fully differentiated myotubes were electrically paced at $14 \mathrm{~V}(1 \mathrm{~Hz})$ for $3 \mathrm{~h} /$ day for 4 consecutive days. After the last bout of contractile activity, myotubes recovered for $21 \mathrm{~h}$ in exosome-depleted differentiation media. Then we collected the conditioned media from both CON and CS myotubes and performed EV isolation using dUC. Morphological analysis using TEM revealed that myotubes released round-shaped vesicles of about 30$100 \mathrm{~nm}$ with intact double lipid layer, indicating presence of sEVs (Fig. 3A). The average size of CS-EVs $(132 \mathrm{~nm})$ as determined by DLS was $\sim 26 \%$ smaller than CON-EVs $(178 \mathrm{~nm})(\mathrm{N}=8, \mathrm{p}<0.05$, Fig. 3B). A twoway repeated measures ANOVA on size distribution showed a significant main interaction effect for CON-EVs and CS-EVs. Multiple comparision analysis showed that CS-EVs had a 3.1-fold higher yield of 100-150 nm sized sEVs than CON-EVs (N=8, Fig. 3C). Conversely, CON-EVs were enriched with an 80fold higher expression of 250-300 nm sized EVs vs. CS-EVs (N=8, Fig. 3C). Overall, our results indicate that chronic stimulation of myotubes induces a shift from $\mathrm{m} / \mathrm{lEVs}$ to primarily sEVs. Finally, zeta potential of CS-EVs $(-14.8 \mathrm{mV})$ was $27 \%$ lower than CON-EVs $(-11.6 \mathrm{mV})(\mathrm{N}=8, \mathrm{p}<0.05$, Fig. 3D). There was no difference in EV protein yield between the two groups $(\mathrm{N}=8$, Fig. 3E).

\section{Chronic stimulation alters proteins associated with EV subtypes in isolated EVs and myotubes}

We measured the expression of common protein markers associated with EV subtypes using Western blotting as per MISEV guidelines [4]. These proteins are used extensively as markers of EV origin (e.g. endosomal, ectosomal or components of other double-membraned organelles), size (sEVs or m/lEVs) and purity of isolation (co-isolated non-EV proteins). We evaluated the expression of proteins normally enriched in exosomes or sEVs (CD63, CD81, Tsg101, HSP70 and Flotillin-1, Alix), proteins found in $\mathrm{m} / \mathrm{lEVs}$ (Cyt $\mathrm{C}$ and $\beta$-actin), and a non-EV marker (Apo-A1). Interestingly, CS-EVs showed increased expression of CD81 and HSP70 (N=9, p<0.05, Fig. 4A and 4B), and a trend towards an increase for Tsg101 $(\mathrm{N}=9, \mathrm{p}=0.07$, Fig. $4 \mathrm{~A}$ and $4 \mathrm{~B}$ ) compared to CON-EVs. We found no difference in the expression of Flotillin-1, Alix and ApoA1 between the two groups ( $\mathrm{N}=6-9$, Fig. 4A and 4B). We did not detect any expression of CD63, Cyt $C$ and $\beta$-actin in the $E V$ preparations from either groups ( $N=9$, Fig. $4 A$ and $4 B$ ). 
Overall Skm-EVs were CD81+ and CD63-, which based on a recent paper [40] indicates that they are likely released through the ectosomal pathway of EV biogenesis.

Lastly, we measured the expression of CD63, CD81, Tsg101, HSP70, Flotillin-1, and ApoA1 in CON and CS myotube whole cell lysates. Surprisingly, CS myotubes showed decreased expression of CD63 (by $32 \%$ ) and ApoA1 (by 67\%) compared to $\mathrm{CON}$ myotubes ( $\mathrm{N}=7, \mathrm{p}<0.05$, Fig. 5A and 5B). No differences in the expression of CD81, Tsg101, HSP70 and Flotillin-1 between CON and CS myotubes were observed (N=6-7, Fig. 5A and 5B). 


\section{Discussion}

Here we sought to elucidate the effect of chronic contractile activity on the biophysical properties of EVs relased from skeletal muscle myotubes. To the best of our knowledge, this is the first study to characterize EVs from skeletal muscle following chronic electrical stimulation, a validated model of chronic exercise in vitro [31], [32]. The results demonstrate that chronically stimulated skeletal muscle myotubes release smaller sized, and more small stable EVs than non-stimulated controls. This occured in tandem with an increase in the expression of sEV markers, CD81 and HSP70 in CS-EVs. Neither CONEVs nor CS-EVs expressed Cyt C or $\beta$-actin. Given that Skm-EVs are largely sEVs, $<$ or around $200 \mathrm{~nm}$ in size, this indicates that these EVs are either of plasma membrane or endosomal origin, and originated distant to Golgi, endoplasmic reticulum, mitochondrial or nuclear components as per MISEV [4]. Further, Skm-EVs were CD81+/CD63- indicating they are likely released via an ecotosomal (plasma membrane) pathway, based on exquisite work by the Théry lab published recently [40]. Both CON-EVs and CS-EVs were also packaged with other cytosolic cargo proteins indicative of sEVs (Tsg101, HSP70, Alix, Flotillin1). Overall, our data showed that chronic stimulation modified the biophysical composition of Skm-EVs. We do not know if this changes the recruitment of cargo in CS-EVs, and/or if that alters their downstream biological activity. The upstream signaling cascades involved, and the origin of Skm-EVs as ectosomal vs. endosomal remains to be confirmed.

Previous work on the effect of acute/chronic exercise on skeletal muscle-derived EVs mostly focused on EV preparations made from whole blood (plasma or serum) [23]-[28], [30], [35], [41]-[43]. Whole blood comprise a heterogenous mixture of vesicles derived from platelets, erythrocytes, endothelial cells, leukocytes, and with a comparatively little proportion from skeletal muscle [21], [24], [25]. Given that skeletal muscle accounts for $\sim 40 \%$ of the body mass and is the major organ system involved in muscle contraction during physical activity, it is important to evaluate the impact of contractile activity on the characteristics of EVs from skeletal muscle specifically. Some groups have successfully used a protein marker of skeletal muscle, alpha-Sarcoglycan (SGCA), to distinguish Skm-EVs in whole blood [21]. While the marker is specific for Skm-EVs, there is no guarantee that all EVs released from skeletal muscle would express it. Secondly, it does not distinguish the EV contribution of the contracting skeletal muscle vs. other skeletal muscle fibers at rest. Lastly, the concentration of SGCA+ EVs was quite low in comparision to EVs from other cell types [21]. These challenges preclude an in depth and thorough characterization of the effect of chronic contractile on Skm-EVs specifically, thereby necessitating the use of alternate approaches as exemplified by this current work.

We used electrical pulse stimulation to mimic chronic exercise in myotubes. Electrical pulse stimulation, which has been extensively used, has been shown to effectively mimic the effects of chronic exercise on augmenting mitochondrial biogenesis in vitro [31], [44]. Increased mitochrondrial content/function is an important adaptation of habitual endurance exercise. Our results showed that mitochondrial biogenesis as measured by MitoTracker staining, COX activity and Cyt C (a critical protein in oxidatve phosphorylation) was increased in chronically stimulated myotubes, in line with reporter results by other groups [31], [32], [45], [46]. In general, our results validated that the chronic stimulation 
protocol was effective in mimicing the effects of chronic contractile activity in vitro, and we subsequently used it to interrogate the effects of contractile activity on Skm-EVs.

TEM results showed that the purified EV preparations were enriched with vesicles between 30$100 \mathrm{~nm}$ in size, as expected when dUC is used as the method of isolation for preferential purification of sEVs. This is in line with previous studies that have also used dUC and shown that C2C12 myotubes release sEVs [47]-[50]. In this current study, we found a 26\% decrease in average size of CS-EVs $(\sim 132$ $\mathrm{nm})$ compared to CON-EVs ( $178 \mathrm{~nm})$. Using DLS, size distribution results showed that CS-EVs were more enriched in sEVs between 100-150 nm, while CON EVs were enriched in 200-250 nm sized EVs. The DLS results are based on light scatter experiments and as such carry a degree of uncertainty as larger particles can scatter more light and mask smaller particles. This explains the slight discrepancy in TEMderived size vs. DLS measurements. Using complimentary orthogonal approaches like tunable resistive pulse sensing (TRPS) or microfluidics resistive pulse sensing (MRPS) can provide further confirmation of size data. These results indicate that electrical stimulation of myotubes induces the preferential release of sEVs. Interestingly, this is in line with other studies that have reported that endurance exercise training potentiates the release of sEVs in circulation [26], [29], [30].

Zeta potential is an important indicator of colloidal stability of particles in dispersed systems, such as suspensions, emulsions and colloidal dispersions [51]. All biological membranes including those encapsulating EVs possess a negative surface charge primarily due to the presence of negatively-charged lipids such as phosphatidylserine. Lower (more negative) zeta potential values confer stability, which signifies a greater degree of electrostatic repulsion and less tendency of aggregation or flocculation between particles [52]. Our result revealed that the EVs isolated had negative zeta potential within the range of -5.9 to $-18.6 \mathrm{mV}$, which is within the range previously reported by other groups [53], [54]. Zeta potential values can change depending on the isolation method used. $\mathrm{dUC}$, which is the most commonly used and the gold-standard method of sEV isolation, can cause aggregation and damage EV integrity due to the high speed of centrifugation [55], [56]. So the zeta potential values measured should be seen in the context of the isolation methodology. Interestingly, zeta potential of CS-EVs was $27 \%$ lower (more stable) than CON-EVs. This might indicate that electrical stimulation alters the physicochemical properties of EVs by increasing the surface charge and stability of EVs.

Our results showed no difference in EV protein yield between CS-EVs vs. CON-EVs, and to the best of our knowledge, no other study has measured the effect of chronic contractile activity on total EV protein yield. The studies that have reported an increase in EV protein concentration in humans [35] and rats [27] after exercise utilized acute exercise models. To ascertain the subtype of EVs isolated, we measured the expression of proteins including exosomal markers (CD81, CD63, Tsg101, HSP70, Alix and Flotillin-1), m/l EV markers (Cyt $C$ and $\beta$-actin), and a non-EV marker (Apo-A1) according to MISEV guidelines [4]. Interestingly, we found that CS-EVs had a higher expression of CD81 and HSP70, and trended towards an increase in Tsg101 compared to CON-EVs. Our observations are in line with previous studies that have shown that both acute/chronic exercise training increases EV protein levels of CD81 and HSP70 in mice and humans [24], [25], [30], [57], [58]. We did not find any difference in the expression of Alix and Flotillin-1 between CON-EV and CS-EV. However, most studies that have reported an increase 
in Alix and Flotillin-1 expression employed acute exercise models [24], [25], [57], [59]. The only study that reported enhanced levels of Flotillin-1 after chronic endurance exercise was done in mice, and the discrepancy in our observations $v s$. theirs may be due to the differences in the type of exercise as well as the source of EVs since vesicle preparations were generated from circulation in the paper [30]. Our present study and its findings are unique as to date it is the only specific assessment of Skm-EVs from contracting myotubes, without the confounding presence of non-Skm-EVs, or Skm-EVs from muscles not exercising.

We did not detect any expression of Cyt $C$ and $\beta$-actin in the EV lysates. Cyt $C$ (a mitochondrial protein) has been extensively used as a $\mathrm{m} / \mathrm{l} \mathrm{EV}$ marker [60], [61], and $\beta$-actin (a protein associated with the cytoskeleton) has been shown to be enriched in larger sized vesicles [62], [63]. Altogether our results support the conclusion that Skm-EVs are enriched with sEVs and absence of Cyt $C$ and $\beta$-actin indicates that these Skm-EVs likely originate through the endosomal or ectosomal pathway. However, given that we did not detect any expression of CD63 in our EV preparations either, it can be inferred that Skm-EVs are likely generated at the plasma membrane. This is because CD63 is an important exosomal marker indicative of endosomal biogenesis. To assess the purity of our Skm-EV isolation protocol, we measured the levels of ApoA1, a non-EV lipoprotein marker. It is not surprising that our EV preparation contains ApoA1 since lipoproteins can be co-isolated with sEVs due to the overlap in their size and density [64]. Lipoprotein contamination is commonly detected in EVs isolated via dUC, as previously reported [54], [56]. To reduce the level of lipoprotein contamination, it is advised that researchers use two or more isolation or purification steps, which is an approach we can utilize in future. Our current Skm-EV preparations from both CON and CS myotubes express ApoA1, but encouragingly the expression of this non-EV co-isolate protein is not different between our control and treatment groups.

Lastly, we wanted to determine if chronic contractile activity altered the expression of EV-related proteins in myotubes. To the best of our knowledge, this is the first study to measure the expression of EV markers in skeletal muscle myotubes following chronic electrical stimulation. We measured the expression of CD63, CD81, Tsg101, HSP70 and Flotillin-1, $\beta$-actin and ApoA1 in CON and CS myotube whole cellextracts. We found that CS myotubes expressed less CD63 and ApoA1 protein content compared to CON myotubes. ApoA1 is a major component of high density lipoprotein. Han et al. showed that ApoA1 can stimulate glucose uptake in C2C12 myotubes [65]. Furthermore, previous studies have shown that exercise increases levels of ApoA1 in humans [66], [67]. The discrepancy in results could be the due to the type of exercise/species model used - we used an in vitro model of chronic stimulation using murine cells, whereas others used plasma from humans after weeks of aerobic exercise. CD63, a protein that belongs to the tetraspanin family, is involved in a host of biological processes including the regulation of cell growth, activation, invasion, differentiation, adhesion, membrane fusion, signaling and protein trafficking [68], [69]. Several studies have shown that CD63 in EVs increases with acute/chronic exercise in humans and rodents [25]-[27], [70]. One study reported no difference in CD63 mRNA in skeletal muscle from human after acute exercise [71], but no one had measured CD63 protein in skeletal muscle post-chronic exercise at the time of writing this manuscript. We did not find a difference in CD81, Tsg101, HSP70 and Flotillin-1 between the groups, which was surprising since we saw an increase in CD81 and HSP70 in EVs from CS myotubes. However, our result is similar to a recent study that showed 
no difference in the protein expression of TSG101 and CD81 in skeletal muscle from healthy humans with acute exercise, even though these proteins were increased in circulatory EVs with exercise. This same study also showed an increase in the mRNA levels of TSG101 in the skeletal muscle of prediabetic subjects with exercise [58]. It is possible that with longer exercise duration, intensity, or recovery, we may observe increase in TSG101 expression. It is not clear why CD81 and HS70 are preferentially packaged into sEVs and released after chronic electrical stimulation. CD81 is involved in membrane fusion and protein trafficking [69], while HSP70 is a chaperone protein involved in protein folding and transport [72]. Does chronic contractile activity increase chaperone-mediated cargo assortment into sEVs, and/or enhance the fusion of existing MVB pool with the plasma membrane or promote ectosomal sEV release? These are tantalizing but unanswered questions about the mechanisms of EV release from the skeletal muscle cells after chronic electrical stimulation that warrant further attention.

In summary, the contractile activity model we used in this study helps to circumvent the challenge of using whole blood-based EV preparations to study the role of Skm-EVs. It is an efficient, fast method to induce mitochondrial biogenesis in cells as quickly as four days and interrogate the effect of contractile activity on Skm-EVs biogenesis, properties and downstream biological activiy, without the heterogenous mixture of EVs in the plasma/serum. We showed that chronic contractile activivty of murine myotubes triggered the release of more stable, and smaller sized Skm-EVs, that are CD81+/CD63and enriched with classical sEV marker proteins (Tsg101, HSP70, Alix, Flotillin-1). Based on our results and recent work [40], we believe this indicates Skm-EVs are ectosomally-derived, originating distally from double-membrane bound intracellular organelles (as supported by the absence of Cyt $C$ and $\beta$-actin expression). The findings are also consistent with the previous reports of chronic exercise in altering EV biophysical characteristics. We do not know if the changes in Skm-EV biophysical properties alters the recruitment of luminal cargo into CS-EVs, nor if that in turn modifies the downstream biological activity of Skm-EVs. The upstream signaling cascades involved, and the origin of Skm-EVs as ectosomal vs. endosomal remains to be confirmed. 
Author Contributions: P.O.O., S.S., B.B. and T.M.P performed experiments in the current study, analyzed data, and created figures. P.O.O. and A.S. helped write and revise the manuscript. E.T., A.R.W., H.I.L. and J.W.G. provided technical and theoretical expertise to complete the work. All authors were involved in manuscript revisions. A.S. designed the project, and helped synthesize data, create figures, write, and edit the manuscript. A.S. is the corresponding author and directly supervised the project. All authors have read, edited and agreed to the published version of the manuscript.

Funding: P.O.O. holds a University of Manitoba Graduate Scholarship. B.B. holds a KRM Graduate Scholarship from the Faculty of Kinesiology and Rec Management. T.M.P. was funded by a Postdoctoral Fellowship from Research Manitoba. This research was funded by operating grants from Research Manitoba (UM Project no. 51156), and University of Manitoba (UM Project no. 50711) to A.S.

Conflicts of Interest: All other authors declare no conflict of interest. The funders had no role in the design of the study; in the collection, analyses, or interpretation of data; or in the writing of the manuscript.

Word count (not including title page, abstract, references, figure legends): 5254 


\section{References}

[1] M. Yáñez-Mó et al., "Biological properties of extracellular vesicles and their physiological functions," J. Extracell. vesicles, vol. 4, p. 27066, May 2015, doi: 10.3402/jev.v4.27066.

[2] D. Armstrong and D. E. Wildman, "Extracellular vesicles and the promise of continuous liquid biopsies," J. Pathol. Transl. Med., vol. 52, no. 1, pp. 1-8, Jan. 2018, doi: 10.4132/JPTM.2017.05.21.

[3] K. Sidhom, P. O. Obi, and A. Saleem, "A Review of Exosomal Isolation Methods: Is Size Exclusion Chromatography the Best Option?," Int. J. Mol. Sci. 2020, Vol. 21, Page 6466, vol. 21, no. 18, p. 6466, Sep. 2020, doi: 10.3390/IJMS21186466.

[4] C. Théry et al., “Minimal information for studies of extracellular vesicles 2018 (MISEV2018): a position statement of the International Society for Extracellular Vesicles and update of the MISEV2014 guidelines," J. Extracell. Vesicles, vol. 7, no. 1, p. 1535750, Dec. 2018, doi: 10.1080/20013078.2018.1535750.

[5] A. Safdar, A. Saleem, and M. A. Tarnopolsky, "The potential of endurance exercise-derived exosomes to treat metabolic diseases," Nat. Rev. Endocrinol., vol. 12, no. 9, pp. 504-517, 2016, doi: 10.1038/nrendo.2016.76.

[6] N. Akbar, V. Azzimato, R. P. Choudhury, and M. Aouadi, “Extracellular vesicles in metabolic disease," Diabetologia, vol. 62, no. 12, pp. 2179-2187, 2019, doi: 10.1007/s00125-019-05014-5.

[7] M. C. Martínez and R. Andriantsitohaina, "Extracellular Vesicles in Metabolic Syndrome," Circ. Res., vol. 120, no. 10, pp. 1674-1686, May 2017, doi: 10.1161/CIRCRESAHA.117.309419.

[8] K. Xu et al., "Extracellular vesicles as potential biomarkers and therapeutic approaches in autoimmune diseases," J. Transl. Med., vol. 18, no. 1, pp. 1-8, Dec. 2020, doi: 10.1186/S12967-02002609-0/FIGURES/2.

[9] R. E. Lane, D. Korbie, M. M. Hill, and M. Trau, “Extracellular vesicles as circulating cancer biomarkers: opportunities and challenges," Clin. Transl. Med., vol. 7, no. 1, p. 14, Dec. 2018, doi: 10.1186/S40169-018-0192-7.

[10] E. Barberis et al., "Circulating Exosomes Are Strongly Involved in SARS-CoV-2 Infection," Front. Mol. Biosci., vol. 8, p. 29, Feb. 2021, doi: 10.3389/FMOLB.2021.632290/BIBTEX.

[11] I. K. Herrmann, M. J. A. Wood, and G. Fuhrmann, "Extracellular vesicles as a next-generation drug delivery platform," Nat. Nanotechnol. 2021 167, vol. 16, no. 7, pp. 748-759, Jul. 2021, doi: 10.1038/s41565-021-00931-2.

[12] C. Soekmadji et al., "The future of Extracellular Vesicles as Theranostics - an ISEV meeting report," J. Extracell. Vesicles, vol. 9, no. 1, Jan. 2020, doi: 10.1080/20013078.2020.1809766.

[13] F. Villa, R. Quarto, and R. Tasso, "Extracellular Vesicles as Natural, Safe and Efficient Drug Delivery Systems," Pharmaceutics, vol. 11, no. 11, Nov. 2019, doi: 10.3390/PHARMACEUTICS11110557.

[14] R. G. C., S. Adeel, and A. Zolt, "Running Forward," Circulation, vol. 129, no. 7, pp. 798-810, Feb. 2014, doi: 10.1161/CIRCULATIONAHA.113.001590.

[15] B. K. Pedersen, "The Physiology of Optimizing Health with a Focus on Exercise as Medicine," Annual Review of Physiology, vol. 81. Annual Reviews Inc., pp. 607-627, Feb. 10, 2019, doi: 10.1146/annurev-physiol-020518-114339.

[16] J. Viña, F. Sanchis-Gomar, V. Martinez-Bello, and M. C. Gomez-Cabrera, "Exercise acts as a drug; The pharmacological benefits of exercise," British Journal of Pharmacology, vol. 167, no. 1. WileyBlackwell, pp. 1-12, Sep. 2012, doi: 10.1111/j.1476-5381.2012.01970.x.

[17] M. C. K. Severinsen and B. K. Pedersen, "Muscle-Organ Crosstalk: The Emerging Roles of Myokines," Endocr. Rev., vol. 41, no. 4, pp. 594-609, Aug. 2020, doi: 10.1210/ENDREV/BNAA016.

[18] B. K. Pedersen, "Muscles and their myokines," J. Exp. Biol., vol. 214, no. 2, pp. 337 LP - 346, Jan. 2011, doi: 10.1242/jeb.048074. 
[19] E. Trovato, V. Di Felice, and R. Barone, "Extracellular Vesicles: Delivery Vehicles of Myokines," Front. Physiol., vol. 10, p. 522, May 2019, doi: 10.3389/fphys.2019.00522.

[20] S. Rome, A. Forterre, M. L. Mizgier, and K. Bouzakri, "Skeletal Muscle-Released Extracellular Vesicles: State of the Art," Front. Physiol., vol. 10, no. JUL, p. 929, 2019, doi: 10.3389/FPHYS.2019.00929.

[21] M. Guescini et al., "Muscle Releases Alpha-Sarcoglycan Positive Extracellular Vesicles Carrying miRNAs in the Bloodstream," PLoS One, vol. 10, no. 5, May 2015, doi: 10.1371/JOURNAL.PONE.0125094.

[22] J. P. Nederveen, G. Warnier, A. Di Carlo, M. I. Nilsson, and M. A. Tarnopolsky, "Extracellular Vesicles and Exosomes: Insights From Exercise Science," Front. Physiol., vol. 11, p. 1757, Feb. 2021, doi: 10.3389/FPHYS.2020.604274/BIBTEX.

[23] M. Whitham et al., "Extracellular Vesicles Provide a Means for Tissue Crosstalk during Exercise," Cell Metab., vol. 27, pp. 237-251, Jan. 2018, doi: 10.1016/j.cmet.2017.12.001.

[24] C. Frühbeis, S. Helmig, S. Tug, and P. Simon \& Eva-Maria Krämer-Albers, "Physical exercise induces rapid release of small extracellular vesicles into the circulation," J. Extracell. Vesicles, 2015, doi: $10.3402 /$ jev.v4.28239.

[25] A. Brahmer et al., "Platelets, endothelial cells and leukocytes contribute to the exercise-triggered release of extracellular vesicles into the circulation," J. Extracell. Vesicles, vol. 8, p. 1615820, Dec. 2019, doi: 10.1080/20013078.2019.1615820.

[26] Y. Bei et al., "Exercise-induced circulating extracellular vesicles protect against cardiac ischemiareperfusion injury," Basic Res. Cardiol., vol. 112, no. 4, p. 38, Jul. 2017, doi: 10.1007/S00395-017-0628Z.

[27] G. P. Oliveira et al., "Effects of acute aerobic exercise on rats serum extracellular vesicles diameter, concentration and small RNAs content," Front. Physiol., vol. 9, no. MAY, p. 532, May 2018, doi: 10.3389/FPHYS.2018.00532/FULL.

[28] Z. Hou et al., “Longterm Exercise-Derived Exosomal miR-342-5p: A Novel Exerkine for Cardioprotection," Circ. Res., vol. 124, no. 9, pp. 1386-1400, Apr. 2019, doi: 10.1161/CIRCRESAHA.118.314635.

[29] C. Ma et al., "Moderate Exercise Enhances Endothelial Progenitor Cell Exosomes Release and Function," Med. Sci. Sports Exerc., vol. 50, no. 10, pp. 2024-2032, Oct. 2018, doi: 10.1249/MSS.0000000000001672.

[30] P. Chaturvedi, A. Kalani, I. Medina, A. Familtseva, and S. C. Tyagi, “Cardiosome mediated regulation of MMP9 in diabetic heart: role of mir29b and mir455 in exercise," J. Cell. Mol. Med., vol. 19, no. 9, pp. 2153-2161, Sep. 2015, doi: 10.1111/JCMM.12589.

[31] G. Uguccioni and D. A. Hood, "The importance of PGC-1 $\alpha$ in contractile activity-induced mitochondrial adaptations," Am. J. Physiol. - Endocrinol. Metab., vol. 300, no. 2, pp. 361-371, Feb. 2011, doi: 10.1152/ajpendo.00292.2010.

[32] N. Nikolić et al., "Electrical Pulse Stimulation of Cultured Human Skeletal Muscle Cells as an In Vitro Model of Exercise," PLoS One, vol. 7, no. 3, p. e33203, Mar. 2012, doi: 10.1371/JOURNAL.PONE.0033203.

[33] C. Théry, S. Amigorena, G. Raposo, and A. Clayton, "Isolation and Characterization of Exosomes from Cell Culture Supernatants and Biological Fluids," Curr. Protoc. Cell Biol., vol. 30, no. 1, Mar. 2006, doi: 10.1002/0471143030.cb0322s30.

[34] C. Zhao and C. Yang, "Nonlinear Smoluchowski velocity for electroosmosis of Power-law fluids over a surface with arbitrary zeta potentials," Electrophoresis, vol. 31, no. 5, pp. 973-979, Mar. 2010, doi: 10.1002/ELPS.200900564.

[35] T. M. Pierdoná et al., "Extracellular vesicles as predictors of individual response to exercise training in youth living with obesity," bioRxiv, p. 2020.11.20.390872, Nov. 2020, doi: 


\subsection{1/2020.11.20.390872.}

[36] W. Mughal et al., "A conserved MADS-box phosphorylation motif regulates differentiation and mitochondrial function in skeletal, cardiac, and smooth muscle cells," Cell Death Dis. 2015 610, vol. 6, no. 10, pp. e1944-e1944, Oct. 2015, doi: 10.1038/cddis.2015.306.

[37] A. Saleem et al., "Polymerase gamma mutator mice rely on increased glycolytic flux for energy production," Mitochondrion, vol. 21, pp. 19-26, 2015, doi: https://doi.org/10.1016/j.mito.2014.12.001.

[38] A. Saleem, P. J. Adhihetty, and D. A. Hood, "Role of p53 in mitochondrial biogenesis and apoptosis in skeletal muscle," https://doi.org/10.1152/physiolgenomics.90346.2008, vol. 37, no. 1, pp. 58-66, Mar. 2009, doi: 10.1152/PHYSIOLGENOMICS.90346.2008.

[39] I. Irrcher, P. Adhihetty, T. Sheehan, A.-M. Joseph, and D. Hood, "PPARgamma coactivator-1alpha expression during thyroid hormone- and contractile activity-induced mitochondrial adaptations," Am. J. Physiol. Cell Physiol., vol. 284, pp. C1669-77, Jun. 2003, doi: 10.1152/ajpcell.00409.2002.

[40] M. Mathieu et al., "Specificities of exosome versus small ectosome secretion revealed by live intracellular tracking of CD63 and CD9," Nat. Commun. 2021 121, vol. 12, no. 1, pp. 1-18, Jul. 2021, doi: 10.1038/s41467-021-24384-2.

[41] J. A. C. Lovett, P. J. Durcan, and K. H. Myburgh, “Investigation of Circulating Extracellular Vesicle MicroRNA Following Two Consecutive Bouts of Muscle-Damaging Exercise," Front. Physiol., vol. 9, no. AUG, Aug. 2018, doi: 10.3389/FPHYS.2018.01149.

[42] G. Annibalini et al., "Muscle and systemic molecular responses to a single flywheel based isoinertial training session in resistance-trained men," Front. Physiol., vol. 10, no. MAY, p. 554, 2019, doi: 10.3389/FPHYS.2019.00554/FULL.

[43] A. E. Rigamonti et al., "Effects of an acute bout of exercise on circulating extracellular vesicles: tissue-, sex-, and BMI-related differences," Int. J. Obes. (Lond)., vol. 44, no. 5, pp. 1108-1118, May 2020, doi: 10.1038/S41366-019-0460-7.

[44] J. Tarum, M. Folkesson, P. J. Atherton, and F. Kadi, “Electrical pulse stimulation: an in vitro exercise model for the induction of human skeletal muscle cell hypertrophy. A proof-of-concept study," Exp. Physiol., vol. 102, no. 11, pp. 1405-1413, Nov. 2017, doi: 10.1113/EP086581.

[45] Y. Tamura, K. Kouzaki, T. Kotani, and K. Nakazato, "Electrically stimulated contractile activityinduced transcriptomic responses and metabolic remodeling in $\mathrm{C} 2 \mathrm{C} 12$ myotubes: twitch vs. tetanic contractions," Am. J. Physiol. Cell Physiol., vol. 319, no. 6, pp. C1029-C1044, Dec. 2020, doi: 10.1152/AJPCELL.00494.2019.

[46] N. Burch et al., "Electric Pulse Stimulation of Cultured Murine Muscle Cells Reproduces Gene Expression Changes of Trained Mouse Muscle," PLoS One, vol. 5, no. 6, p. e10970, 2010, doi: 10.1371/JOURNAL.PONE.0010970.

[47] A. Forterre et al., "Myotube-derived exosomal miRNAs downregulate Sirtuin1 in myoblasts during muscle cell differentiation," Cell Cycle, vol. 13, no. 1, p. 78, Jan. 2014, doi: 10.4161/CC.26808.

[48] R. D. Madison, C. McGee, R. Rawson, and G. A. Robinson, "Extracellular vesicles from a muscle cell line (C2C12) enhance cell survival and neurite outgrow th of a motor neuron cell line (NSC34)," J. Extracell. Vesicles, vol. 3, no. 1, pp. 1-9, 2014, doi: 10.3402/JEV.V3.22865.

[49] H. Sork et al., "Heterogeneity and interplay of the extracellular vesicle small RNA transcriptome and proteome," Sci. Reports 2018 81, vol. 8, no. 1, pp. 1-12, Jul. 2018, doi: 10.1038/s41598-018-284859.

[50] D. P. Romancino et al., "Identification and characterization of the nano-sized vesicles released by muscle cells," FEBS Lett., vol. 587, no. 9, p. 1379, May 2013, doi: 10.1016/J.FEBSLET.2013.03.012.

[51] G. Midekessa et al., "Zeta Potential of Extracellular Vesicles: Toward Understanding the Attributes that Determine Colloidal Stability," ACS Omega, vol. 5, no. 27, pp. 16701-16710, Jul. 2020, doi: 10.1021/ACSOMEGA.0C01582/SUPPL_FILE/AO0C01582_SI_003.AVI.

[52] E. Beit-Yannai, S. Tabak, and W. D. Stamer, "Physical exosome:exosome interactions," J. Cell. Mol. 
Med., vol. 22, no. 3, pp. 2001-2006, Mar. 2018, doi: 10.1111/JCMM.13479.

[53] I. Helwa et al., "A Comparative Study of Serum Exosome Isolation Using Differential Ultracentrifugation and Three Commercial Reagents," PLoS One, vol. 12, p. e0170628, Jan. 2017, doi: 10.1371/journal.pone.0170628.

[54] M. C. Deregibus et al., "Charge-based precipitation of extracellular vesicles," Int. J. Mol. Med., vol. 38, no. 5, p. 1359, Nov. 2016, doi: 10.3892/IJMM.2016.2759.

[55] R. Linares, S. Tan, C. Gounou, N. Arraud, and A. R. Brisson, "High-speed centrifugation induces aggregation of extracellular vesicles," J. Extracell. vesicles, vol. 4, p. 29509, Dec. 2015, doi: 10.3402/jev.v4.29509.

[56] K. W. Witwer et al., "Standardization of sample collection, isolation and analysis methods in extracellular vesicle research," J. Extracell. vesicles, vol. 2, p. 10.3402/jev.v2i0.20360, May 2013, doi: 10.3402/jev.v2i0.20360.

[57] S. Helmig, C. Frühbeis, E. M. Krämer-Albers, P. Simon, and S. Tug, "Release of bulk cell free DNA during physical exercise occurs independent of extracellular vesicles," Eur. J. Appl. Physiol., vol. 115, no. 11, pp. 2271-2280, Nov. 2015, doi: 10.1007/S00421-015-3207-8.

[58] G. Warnier et al., "Effects of an acute exercise bout in hypoxia on extracellular vesicle release in healthy and prediabetic subjects," https://doi.org/10.1152/ajpregu.00220.2021, vol. 322, no. 2, pp. R112-R122, Feb. 2022, doi: 10.1152/AJPREGU.00220.2021.

[59] R. Barone et al., "Skeletal muscle Heat shock protein 60 increases after endurance training and induces peroxisome proliferator-activated receptor gamma coactivator $1 \alpha 1$ expression," Sci. Rep., vol. 6, Jan. 2016, doi: 10.1038/SREP19781.

[60] Y. Yoshioka, Y. Konishi, N. Kosaka, T. Katsuda, T. Kato, and T. Ochiya, "Comparative marker analysis of extracellular vesicles in different human cancer types," J. Extracell. Vesicles, vol. 2, no. 1, 2013, doi: 10.3402/JEV.V2I0.20424.

[61] L. A. Arteaga-Blanco et al., "Characterization and internalization of small extracellular vesicles released by human primary macrophages derived from circulating monocytes," PLoS One, vol. 15, no. 8, p. e0237795, Aug. 2020, doi: 10.1371/JOURNAL.PONE.0237795.

[62] S. La Shu et al., "Purity and yield of melanoma exosomes are dependent on isolation method," J. Extracell. Vesicles, vol. 9, no. 1, Jan. 2020, doi: 10.1080/20013078.2019.1692401/SUPPL_FILE/ZJEV_A_1692401_SM9173.ZIP.

[63] C. Tucher et al., "Extracellular vesicle subtypes released from activated or apoptotic Tlymphocytes carry a specific and stimulus-dependent protein cargo," Front. Immunol., vol. 9, no. MAR, p. 534, Mar. 2018, doi: 10.3389/FIMMU.2018.00534/BIBTEX.

[64] N. Karimi et al., "Detailed analysis of the plasma extracellular vesicle proteome after separation from lipoproteins," Cell. Mol. Life Sci., vol. 75, no. 15, p. 2873, Aug. 2018, doi: 10.1007/S00018-0182773-4.

[65] R. Han et al., "Apolipoprotein A-I stimulates AMP-activated protein kinase and improves glucose metabolism," Diabetologia, vol. 50, no. 9, pp. 1960-1968, Sep. 2007, doi: 10.1007/S00125-007-0752-7.

[66] A. Tofighi, F. Rahmani, B. J. Qarakhanlou, and S. Babaei, "The Effect of Regular Aerobic Exercise on Reverse Cholesterol Transport A1 and Apo Lipoprotein A-I Gene Expression in Inactive Women," Iran. Red Crescent Med. J., vol. 17, no. 4, p. 26321, Apr. 2015, doi: 10.5812/IRCMJ.17(4)2015.26321.

[67] R. Yazdani, H. Marefati, A. Shahesmaeili, S. Nakhaei, A. Bagheri, and M. Dastoorpoor, "Effect of Aerobic Exercises on Serum Levels of Apolipoprotein A1 and Apolipoprotein B, and Their Ratio in Patients with Chronic Obstructive Pulmonary Disease," Tanaffos, vol. 17, no. 2, p. 82, 2018, Accessed: Nov. 23, 2021. [Online]. Available:/pmc/articles/PMC6320561/.

[68] M. S. Pols and J. Klumperman, "Trafficking and function of the tetraspanin CD63," Exp. Cell Res., vol. 315, no. 9, pp. 1584-1592, May 2009, doi: 10.1016/J.YEXCR.2008.09.020. 
[69] Z. Andreu and M. Yáñez-Mó, "Tetraspanins in extracellular vesicle formation and function," Front. Immunol., vol. 5, no. SEP, p. 442, 2014, doi: 10.3389/FIMMU.2014.00442/BIBTEX.

[70] K. Bertoldi et al., "Circulating extracellular vesicles in the aging process: impact of aerobic exercise," Mol. Cell. Biochem., vol. 440, no. 1-2, pp. 115-125, Mar. 2018, doi: 10.1007/S11010-0173160-4/FIGURES/8.

[71] R. T. Garner, J. S. Solfest, Y. Nie, S. Kuang, J. Stout, and T. P. Gavin, "Multivesicular body and exosome pathway responses to acute exercise," Exp. Physiol., vol. 105, no. 3, pp. 511-521, Mar. 2020, doi: 10.1113/EP088017.

[72] M. P. Mayer and B. Bukau, "Hsp70 chaperones: Cellular functions and molecular mechanism," Cell. Mol. Life Sci. , vol. 62, no. 6, p. 670, Mar. 2005, doi: 10.1007/S00018-004-4464-6. 


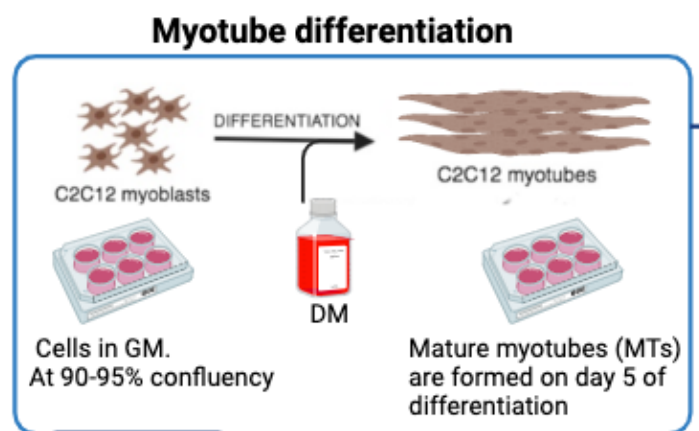

Day 1 - 5

Step 1
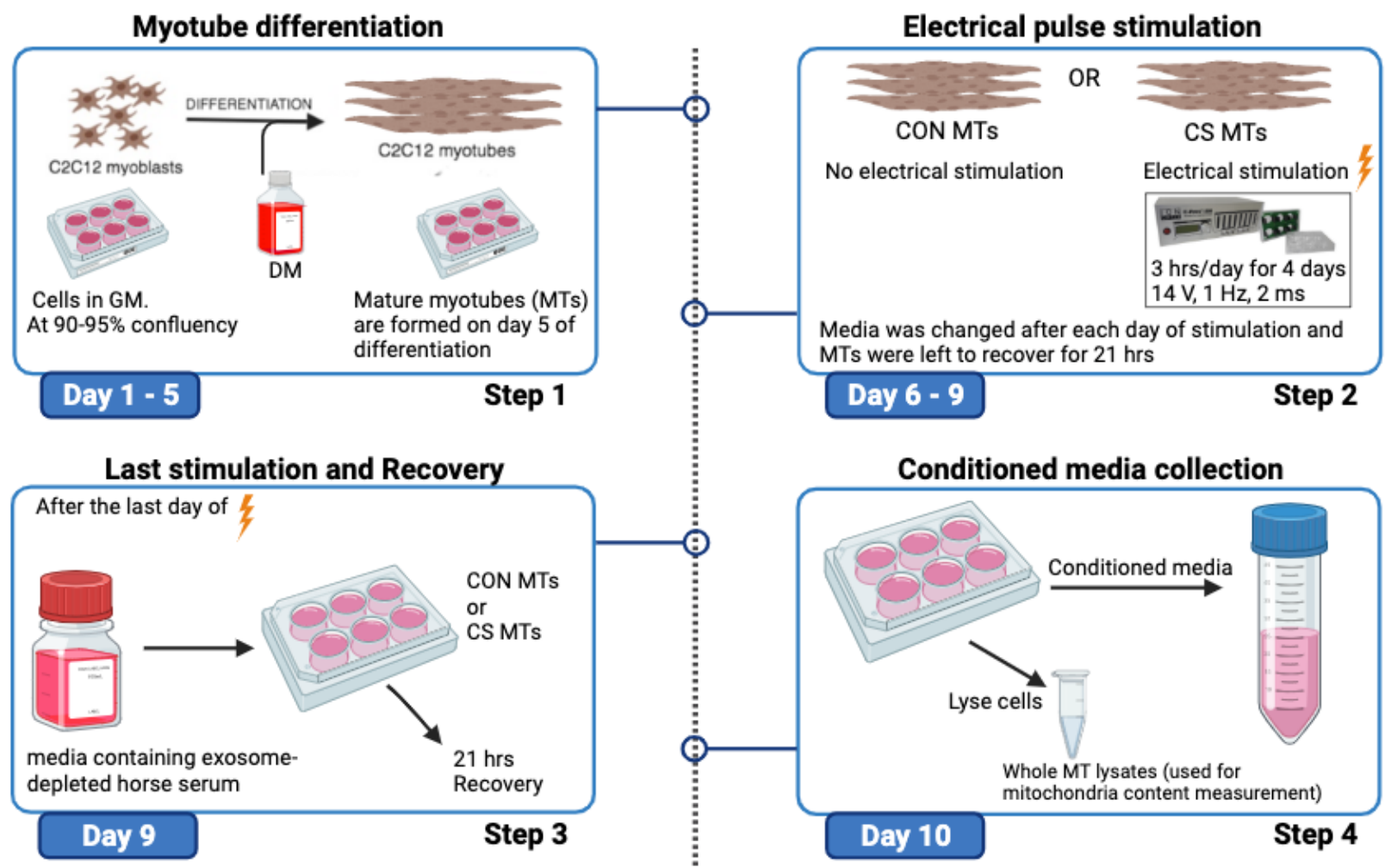

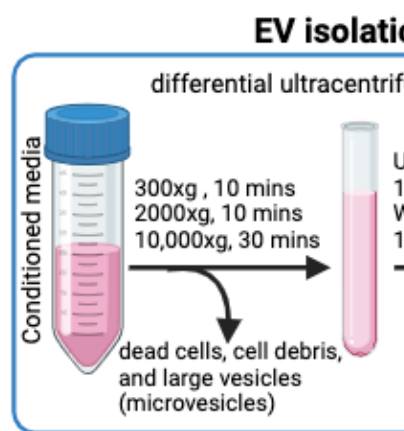

Day 10

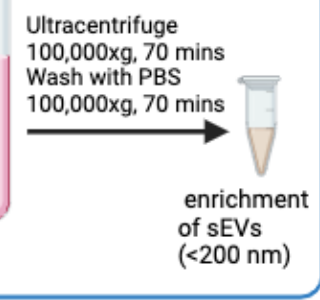

Step 5

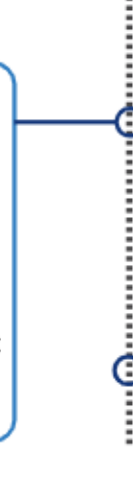

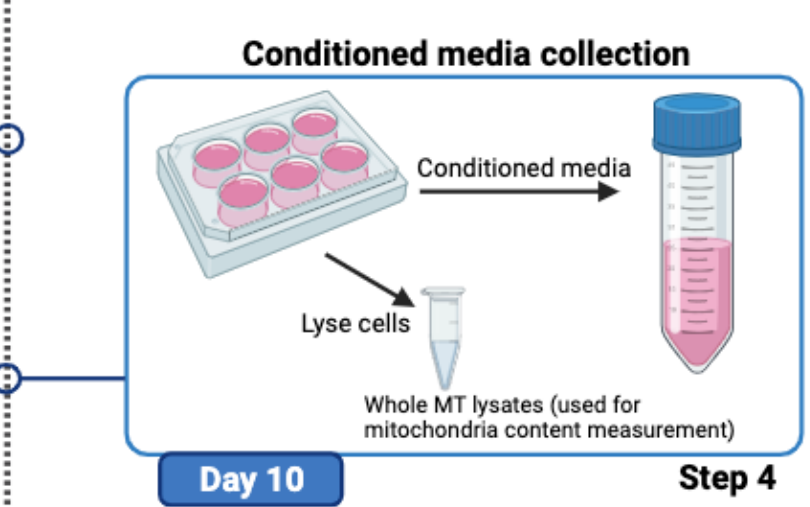

Biophysical analysis

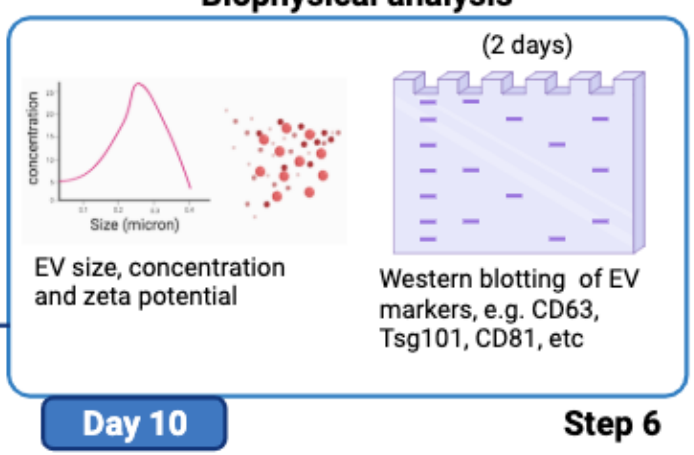

Figure 1. Overall experimental design. C2C12 myoblasts were fully differentiated into myotubes (MTs). MTs were divided into control (CON) and chronic stimulation (CS) plates. CS MTs were electrically paced for 3h/day x 4 days at $14 \mathrm{~V}$ to mimic chronic endurance exercise in vitro using IonOptix ECM. Media was changed after each day of stimulation and cells were left to recover for $21 \mathrm{~h}$ after each bout of contractile activity. After the last day of stimulation, media was switched to differentiation media with exosome-depleted horse serum in both CON and CS MTs and cells were allowed to recover for $21 \mathrm{~h}$. Conditioned media from control or stimulated myotubes was collected and MT lysates were harvested for mitochondrial biogenesis measurement. EVs were isolated from conditioned media via differential ultracentrifugation (dUC) and characterized by size, zeta potential, concentration, protein yield and markers of EV subtypes in line with MISEV guidelines.

Abbreviations: GM - growth media, DM - differentiation media, sEVs - small EVs 

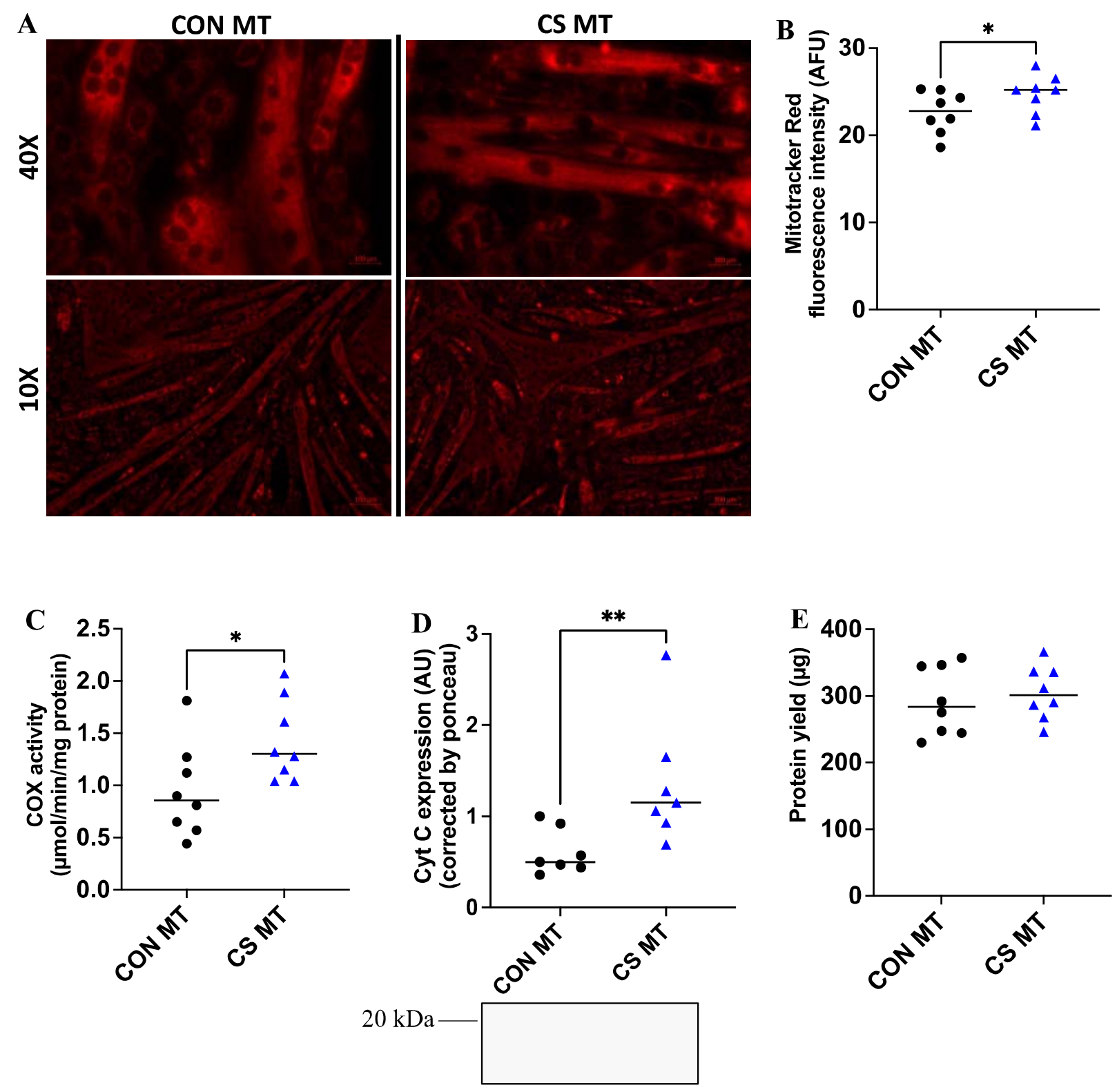

Figure 2. Effect of CS on mitochondrial biogenesis and myotube (MT) protein yield. (A) Representative fluorescent images of CON and CS MTs stained with MitoTracker Red CMXRos (50 nM) at 40X and 10X magnification. Scale bar: $100 \mu \mathrm{m}$. (B) Quantification of MitoTracker Red staining showed 9\% increase in mitochondrial content in CS MTs vs. CON MTs. Images were quantified using imageJ software (NIH, Java 1.8.0_112). (C) Mitochondrial biogenesis measured using cytochrome $c$ oxidase (COX) activity showed $\sim 51 \%$ increase in CS MTs vs. CON MTs. (D) Cytochrome $c$ (Cyt C) was 2.2-fold higher in CS MTs vs. CON MTs. (E) Protein yield was unchanged between groups. Data were analyzed using an unpaired Student's t-test and expressed as scatter plots with mean $\left(\mathrm{N}=7-8,{ }^{*} \mathrm{p}<0.05\right)$. 


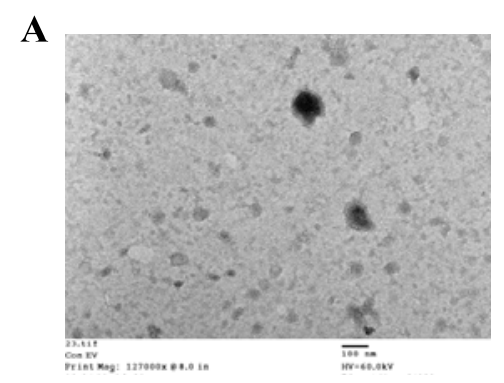

CON EV

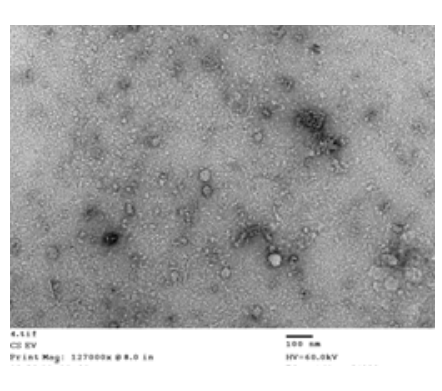

CS EV

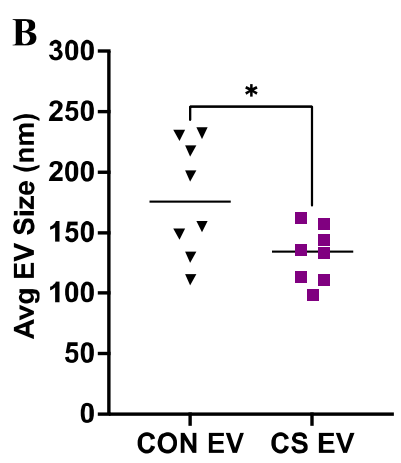

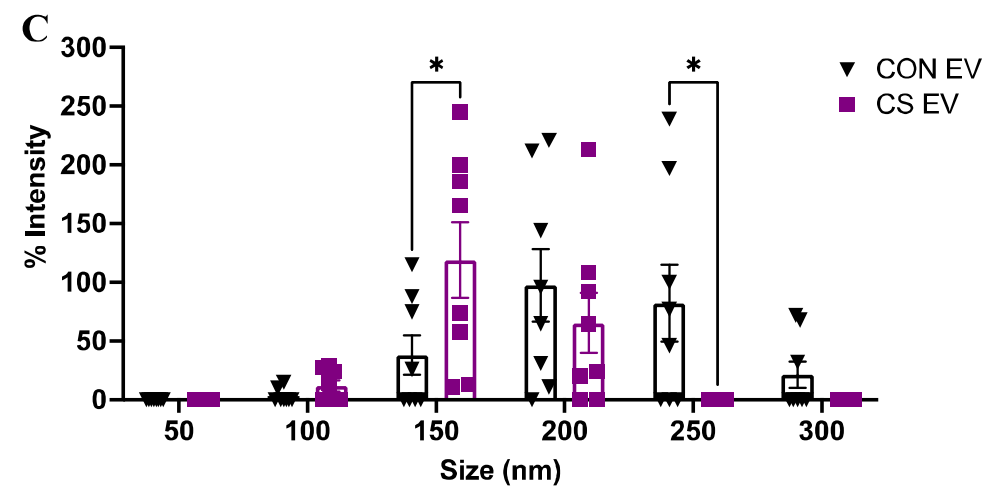
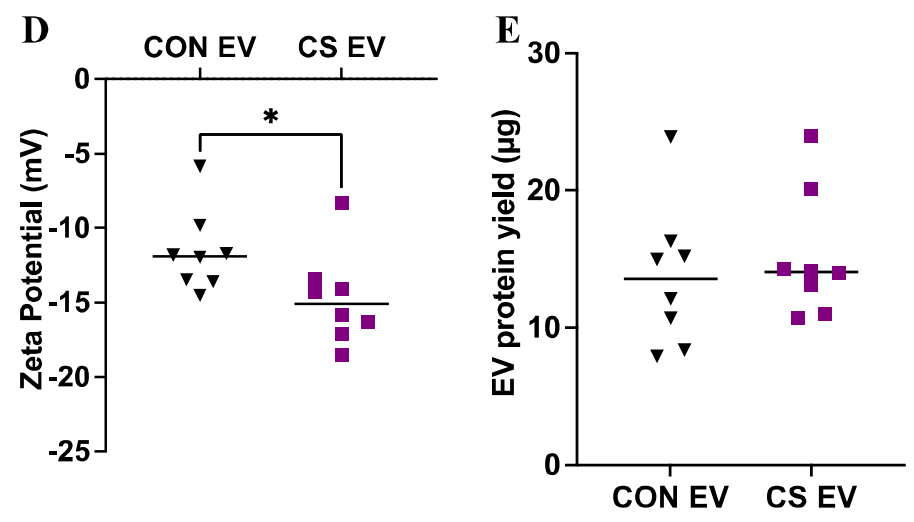

Figure 3. Effect of CS on the biophysical properties of EVs. (A) Representative transmission electron microscopy images of EVs released from the CON and CS myotubes. Arrows indicate the presence of sEVs $\sim 30-100 \mathrm{~nm}$ in diameter; scale bar $=100 \mathrm{~nm}$. (B) Average EV size was $\sim 26 \%$ lower in CS EVs vs. CON EVs $\left(\mathrm{N}=8,{ }^{*} \mathrm{p}<0.05\right)$. (C) EV size distribution showed CS EVs had a3.1-fold higher enrichment of 100-150 $\mathrm{nm}$ sized EVs vs. CON EVs. Conversely, CON EVs were enriched with 200-250 nm sized EVs vs. CS EVs ( $\mathrm{N}=8$, $\left.{ }^{*} \mathrm{p}<0.05\right)$. (D) Zeta potential was $27 \%$ lower in CS EVs vs. CON EVs ( $\mathrm{N}=8$, $\left.{ }^{*} \mathrm{p}<0.05\right)$. (E) EV protein yield remained unchanged between groups. Data were analyzed using an unpaired Student's t-test in panels B, D and E, and by a two-way repeated measures ANOVA in panel C, with multiple comparisions corrected using Sidak's post hoc test. 
A

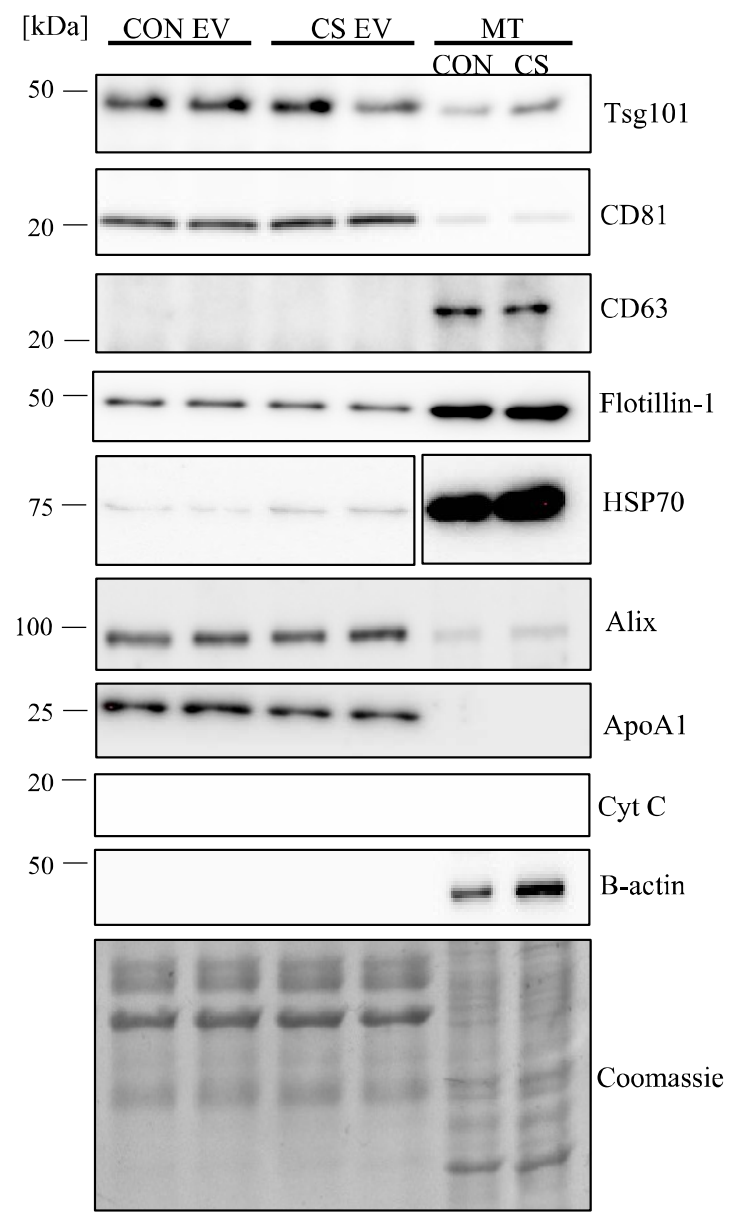

\section{B}
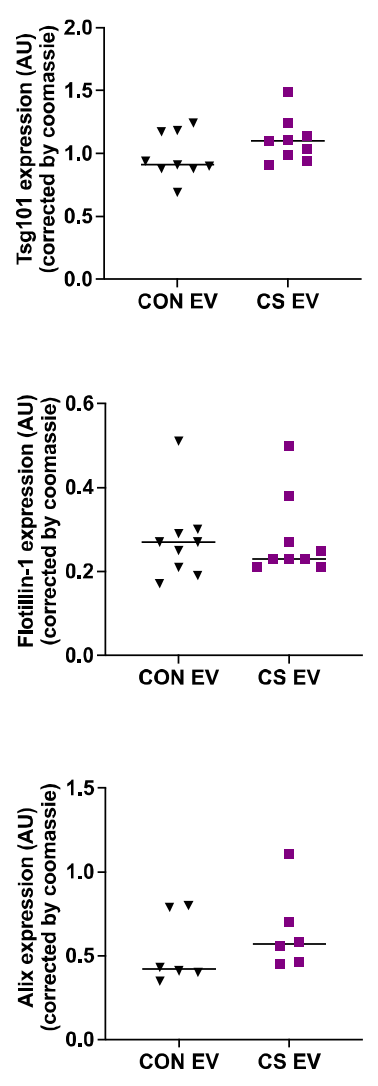
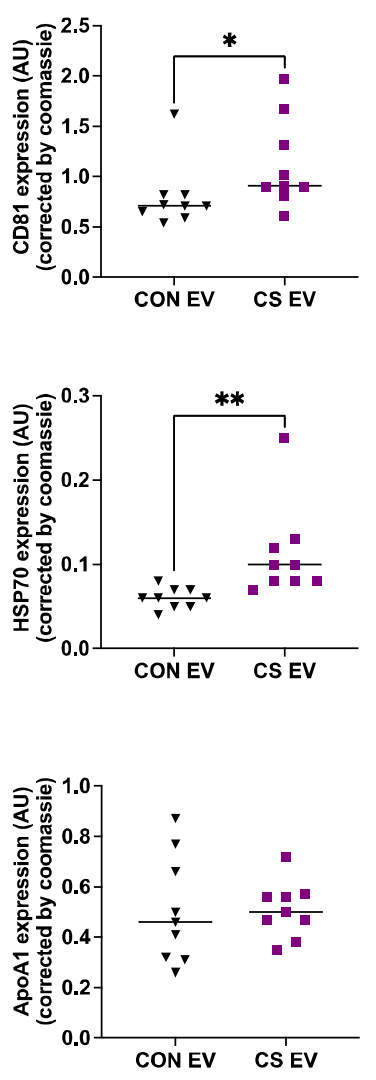

Figure 4. Effect of chronic stimulation on the expression of proteins related to EV subtypes. (A) Equal amounts $(5 \mu \mathrm{g})$ of proteins from CON or CS EVs were subjected to 12\% SDS-PAGE for protein separation and Western blotting analysis. Coomassie blue gel staining was used as a loading control. Expression of sEV markers, CD81 (22 kDa) and HSP70 (70 kDa) was significantly increased in CS EVs by $41 \%$ and $87 \%$, respectively, compared with CON EV. There was no difference in Tsg101 (46 kDa), Flotillin-1 (48 kDa), Alix (90 kDa) and ApoA1 (25 kDa) between groups. Additionally, CD63 (28 kDa), cytochrome c (12 kDa) and beta-actin ( $42 \mathrm{kDa}$ ) were not detected in the the skeletal muscle-derived EV preparations irrespective of stimulation. (B) Quantification of immunoblot analysis from panel A is represented here. Data were analyzed using an unpaired Student's t-test, are expressed as scatter plots with mean, ${ }^{*} \mathrm{p}<0.05,{ }^{* *} \mathrm{p}<0.01$, $\mathrm{N}=6-9$. 
A

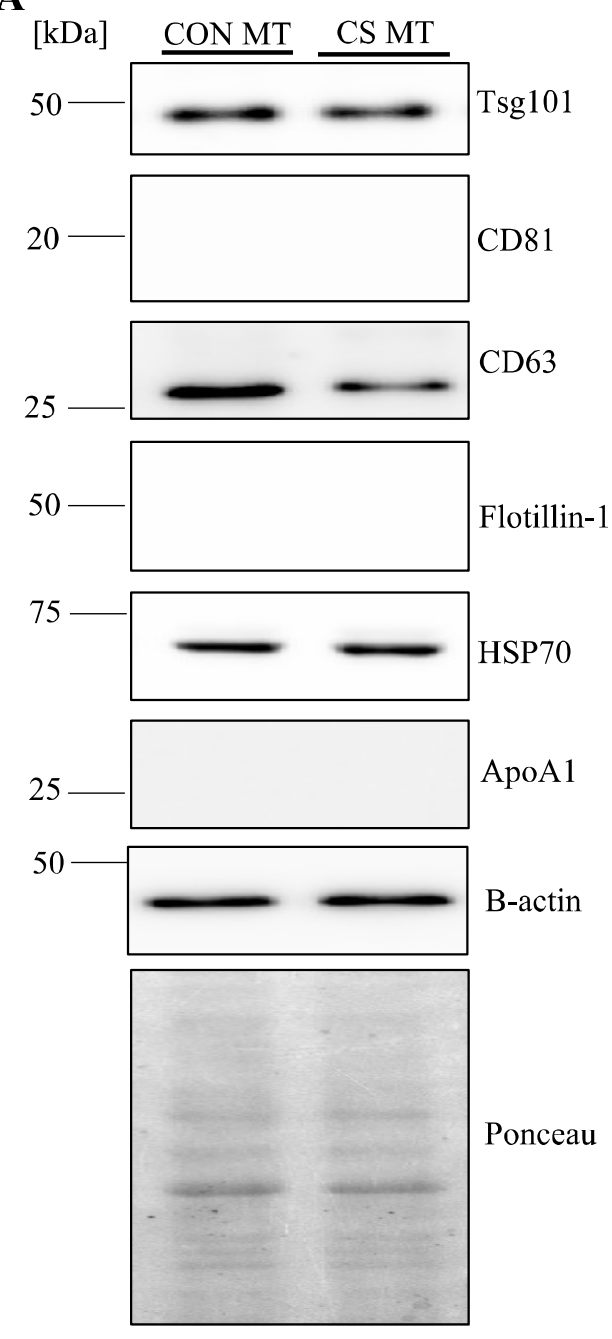

B
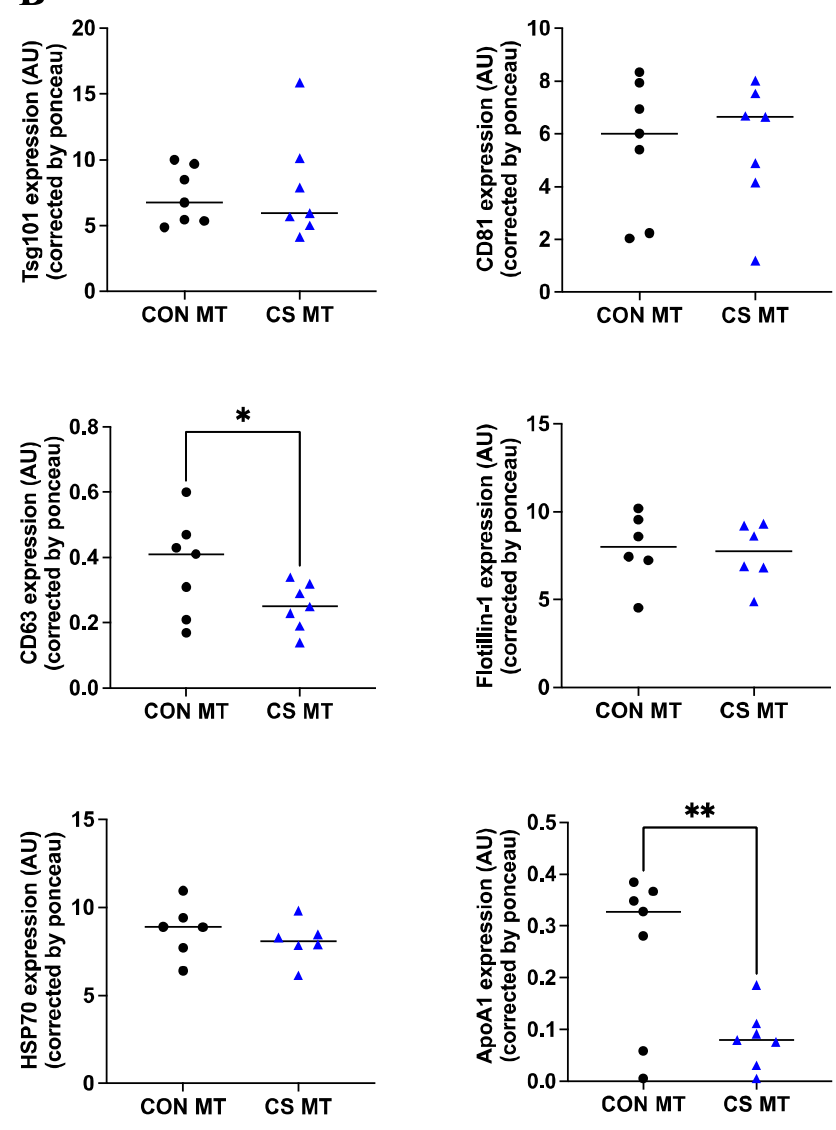

Figure 5. Effect of chronic stimulation on the expression of proteins related to EV subtypes in myotube lysates. (A) Equal amounts ( $5 \mu \mathrm{g}$ ) of proteins from CON or CS myotube extracts were resolved on a $12 \%$ SDS-PAGE for Western blotting analysis. Ponceau was used as a loading control. Protein expression of CD63 (exosomal marker) and ApoA1, was significantly lower in CS MTs compared with CON MTs. There was no difference in levels of Tsg101, CD81, Flotillin-1, HSP70 and Alix between the groups. (B) Quantification of immunoblot analysis from panel A is represented here. Data were analyzed using an unpaired Student's t-test, are expressed as scatter plots with mean, ${ }^{*} \mathrm{p}<0.05,{ }^{* *} \mathrm{p}<0.01, \mathrm{~N}=6-7$. 